\title{
The RKKY interactions and the Mott Transition
}

\author{
G. Moeller ${ }^{1}$, V. Dobrosavljević ${ }^{2}$, and A. E. Ruckenstein ${ }^{1}$ \\ ${ }^{1}$ Serin Physics Laboratory, Rutgers University, Piscataway, NJ 08855-0849 \\ ${ }^{2}$ National High Magnetic Field Laboratory, Florida State University \\ 1800 E. Paul Dirac Dr., Tallahassee, Florida 32306
}

\begin{abstract}
A two-site cluster generalization of the Hubbard model in large dimensions is examined in order to study the role of short-range spin correlations near the metal-insulator transition (MIT). The model is mapped to a two-impurity Kondo-Anderson model in a self-consistently determined bath, making it possible to directly address the competition between the Kondo effect and RKKY interactions in a lattice context. Our results indicate that the RKKY interactions lead to qualitative modifications of the MIT scenario even in the absence of long range antiferromagnetic ordering.
\end{abstract}

PACS numbers: 75.20.Hr, 71.55.Jv

\section{INTRODUCTION}

The competition between the Kondo effect and the RKKY interactions is a recurring theme in many of the most interesting phenomena associated with the physics of strong electronic correlations. When the RKKY interactions predominate, the result is long-range magpetic ordering, as found in many heavy-fermion materials]. In situations where magnetic ordering is absent, the manifestations are more subtle, but often equally fundamental. In particular, it has been suggestedle that this competition lies at the core of the proposed "two-fluid" behavior, and "micromagnetism" found in some non-magnetic heavy fermion systems. Another interesting class of systems where both the Kondo effect and the RKKY correlations are believed to be crucial are exemplified by doped semiconductors 3 near the metal-ipglator transition. In these systems, non-Fermi liquid张 metallic behavior is observed, suggesting the coexistence of local moments and conduction electrons that seem decoupled from each other - another manifestation of the "twofluid" behaviond. There are many further examples where these effects are of key importance. Unfortunately, there are very few theoretical approaches that are able to treat both the Kondo physics and the RKKY correlations on the same footing and provide a convincing picture of these interesting phenomena.

Theoretically, much of our current understanding of strongly correlated metallic phases relies on a variety of mean-field descriptions, most of which essentially emphasize the Kondo aspect of the problem. Several approaches have been proposed, but the most elaborate one, combining many of the pre-existing ideas in the field, is based on taking the limit of large spatial dimensionality This method represents a generalization 8 of simple, but

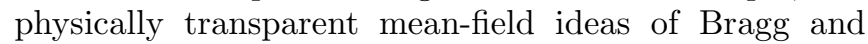
Williamsl, as applied to interacting electronic systems. In this-picture, the electron residing on a given site is viewed 10 as a Kondo spin which is coupled by an exchange interaction to a bath consisting of the remaining electrons. Formally, the problem is mapped 10 onto an Anderson impurity model supplemented by an additional self-consistency condition. The Kondo resonance of the impurity model maps te the heavy quasiparticle band, and the Mott transition 114 is driven by the vanishing of the Kondo temperature $T_{\text {Kondo }} \sim m / m^{*}$. Besides providing an appealing, physically transparent picture of the correlated state and the metal-insulator transition, the $d=\infty$ method provides a quantitatively accurate computational approach valid in the entire temperature range. As it treats at the same level both the coherent, quasiparticle excitations, and the incoherent collective inelastic processes, the method even allows for a description of fully incoherent, non-Fermi liquid metallic states 15 .

In spite of the successes of the $d=\infty$ mean-field approach, it remains unable to address several important physical questions. Since it based on a mapping on a single-site (impurity) model, it cannot properly account for the competition between the Kondo effect and the spin-spin correlations between neighboring sites - the effect that we have argued is crucial in a number of physical situations. The locality inherent in this formulation leads to another feature that is likely to be an artifact of mean-field theory: the "pinning" of the density of states at the Fermi level16. More precisely, this effect can be directly traced to the momentum independence of the local self-energy, reflecting the lack of spatial correlations. In the context of strongly correlated, but weakly disordered system 17 , the pinning condition was shown to result in a discontinuous jump of the DC conductivity at $T=0$ - the minimum metallic conductivity. If the pinning is relaxed, it is conceivable that a continuous behavior of the conductivity would follow, thus qualitatively modifying our picture of transport near the metalinsulator transition 3 .

In order to address the limitations of the existing $d=\infty$ theory, a most straightforward approach would be to investigate systematic $1 / d$ corrections resulting from finite dimensionality. Several different methods for performing such expansions have been proposed 17.18 , but each of these approaches result in formidable technical difficulties, making it difficult to address the finite dimensional effects in a simple and elegant fashion. In this paper, we take an alternative route: we propose to extend the existing theories in $d=\infty$ in a way that mimics the most important physical effects of finite dimension- 
ality. Given the fact that the general large-dimensions philosophy is based on the mapping of a lattice models onto appropriate impurity models, the appropriate impurity model displaying the relevant physics is the two impurity Kondo (Anderson) model19, which is often used as a simplest model for the study of the RKKY-Kondo competition. Using standard methods $\mathbf{3}$, we can obtain a lattice version of this model by self-consistently embedding it in an appropriate medium. The resulting model is the "minimum model" that allows us to go beyond the limitations imposed by the conventional $d=\infty$ approach, without performing uncontrolled or unjustified approximations.

In the rest of this paper, we define and examine this model, and indicate how the new features inherent to the RKKY-Kondo competition modif the standard $d=\infty$ results for the Hubbard model11 14 . Specifically, we investigate the modifications of the Mott transition in a single-band Hubbard model at half filling. We conclude that the RKKY interactions represent a relevant perturbation, relaxing the pinning condition and qualitatively modifying the nature of the metal-insulator transition.

\section{THE MODEL}

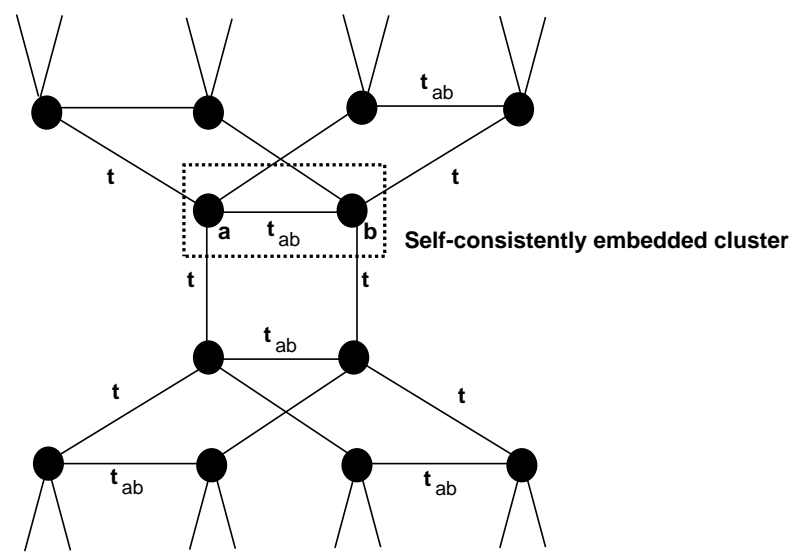

FIG. 1. Lattice structure of the doubled Bethe lattice and effective two impurity cluster.

We begin our discussion by defining the model that we consider, and derive the corresponding self-consistency conditions by performing the $d \rightarrow \infty$ limit. While the limit of infinite dimensions does not impose any restrictions on the lattice structure studied 8 , the equations become particularly simple and easy to derive in the case of a Bethe lattice 12, 13, t. The qualitative features of the model will be identical as on other lattices, and the resulting spectral functions are closer to the three dimensional situation than for example on the $d=\infty$ hypercubic lattice. The "minimum model" that we propose is then obtained by doubling the Bethe lattice (with hopping $t$ ), and allowing the electrons to hop between the Bethe lattices with hopping $t_{a b}$. The geometry of the resulting lattice is shown (for coordination number $z=3$ ) in Fig. (11).

Denoting the creation operators corresponding to the two Bethe lattices with $a_{\sigma}^{\dagger}$ and $b_{\sigma}^{\dagger}$, the Hamiltonian can be written as

$$
\begin{aligned}
H & =-t \sum_{<i, j>, \sigma}\left(a_{i \sigma}^{\dagger} a_{j \sigma}+b_{i \sigma}^{\dagger} b_{j \sigma}\right)+t_{a b} \sum_{i \sigma}\left(a_{i \sigma}^{\dagger} b_{i \sigma}+h . c .\right) \\
& +U \sum_{i \sigma}\left(n_{a i \sigma} n_{a i-\sigma}+n_{b i \sigma} n_{b i-\sigma}\right)-\mu \sum_{i \sigma}\left(n_{a i \sigma}+n_{b i \sigma}\right)
\end{aligned}
$$

where $U$ is the Coulomb potential and $t$ is the nearestneighbor hopping amplitude; $t_{a b}$ is the hopping amplitude between the two lattices.

It should be stressed at this point that this model clearly breaks translational invariance by singling out pairs of sites connected by hopping elements $t_{a b}$. While this feature appears somewhat artificial in a uniform system in which all neighbors are equivalent, it leads to a coptrolled and non-trivial modification of the $d=\infty$ limit20. In contrast to the standard single-band Hubbard model in infinite dimensions, in which electrons solely undergo temporal fluctuations, our model also allows fer spatial fluctuations. A systematic expansion in $1 / d 17,20$ includes exactly these processes and the model can therefore be interpreted as including some of the effects of finite dimensionality.

It is clear that the model by construction enables us to study nearest-neighbor spin-correlations. In physical terms, for $t_{a b}$ large, the model favors the formation of singlet pairs (dimers) from the "a-b" sites. Interestingly, this symmetry breaking is not unreasonable in disordered systems, where each site $a$ has another "preferred" neighboring site $b$, with which dimerization will be favored. This notion is at the heart of the "random singlet" ordering of Bhatt and Lee21, describing the singular thermodynamics of doped semiconductors. Notice, however, that a variety of additional interpretations is possible. In particular, the model may alternatively viewed as a two band model or as two coupled layers22.

As usual, the problem simplifies considerably in the large coordination (large dimension) limit, where a mapping to an appropriate impurity model is obtained. Using standard methods 8 , we proceed by rescaling the hopping amplitude $t$ as $t \rightarrow \frac{t}{\sqrt{m}}(m=z-1$ is the "branching ratio" of the Bethe lattice), and taking the limit $m \rightarrow \infty$. The result is an effective two-impurity Anderson model embedded in a self-consistently determined bath. We introduce spinors $\mathbf{c}_{\sigma}^{*}(\tau)=\left(a_{\sigma}^{*}(\tau), b_{\sigma}^{*}(\tau)\right)$ and the matrix Green function

$$
\mathbf{G}_{\sigma}\left(\tau-\tau^{\prime}\right)=\left(\begin{array}{cc}
-\left\langle\mathcal{T} a_{\sigma} \tau a_{\sigma} \tau^{\prime \dagger}\right\rangle & -\left\langle\mathcal{T} a_{\sigma}(\tau) b_{\sigma}^{\dagger}\left(\tau^{\prime}\right)\right\rangle \\
-\left\langle\mathcal{T} b_{\sigma}(\tau) a_{\sigma}^{\dagger}\left(\tau^{\prime}\right)\right\rangle & -\left\langle\mathcal{T} b_{\sigma}(\tau) b_{\sigma}^{\dagger}\left(\tau^{\prime}\right)\right\rangle
\end{array}\right)
$$

with

$$
\mathbf{G}\left(i \omega_{n}\right)=-\int_{0}^{\beta} e^{i \omega_{n} \tau}\left\langle T_{\tau} \mathbf{c}(\tau) \mathbf{c}^{\dagger}(0)\right\rangle_{S_{e f f}}
$$


Notice that due to spin conservation $\mathbf{G}_{\sigma}=\delta_{\sigma \sigma^{\prime}} \mathbf{G}_{\sigma \sigma^{\prime}}$.

The effective action can then be written in matrix form as

$$
\begin{aligned}
S_{e f f}\left[\mathbf{c}_{\sigma}, \mathbf{c}_{\sigma}^{*}\right]= & -\sum_{\sigma} \sum_{i \omega_{n}} \mathbf{c}_{\sigma \mathbf{n}}^{*} \mathbf{G}_{\mathbf{0}}^{-\mathbf{1}}\left(i \omega_{n}\right) \mathbf{c}_{\sigma \mathbf{n}} \\
& +U \int_{0}^{\beta} d \tau\left(n_{a \uparrow} n_{a \downarrow}+n_{b \uparrow} n_{b \downarrow}\right)
\end{aligned}
$$

where the self-consistency condition reads

$$
\mathbf{G}_{\mathbf{0}}^{-\mathbf{1}}\left(i \omega_{n}\right)=\left(\begin{array}{cc}
i \omega_{n}+\mu & -t_{a b} \\
-t_{a b} & i \omega_{n}+\mu
\end{array}\right)-t^{2} \mathbf{G}\left(\mathbf{i} \omega_{\mathbf{n}}\right)
$$

While solving this model for general values of the parameters represents a highly nontrivial task, we immediately recognize some well known limiting cases. In the limit $t_{a b}=0$, the two Bethe lattices decouple, and the model reduces to the well known single-band Hubbard model in $d=\infty 1144$. At half filling, this model undergoes a Mott transition at $U=U_{c_{2}}$, which is preceded by a formation of a pseudo-gap and the coexistence 12,23 of a metallic and an insulating solution in the region $U=U_{c_{1}}<U<U=U_{c_{2}}$. However, the metallic solution is lower in energy 23 at $T=0$ throughout the coexistence region, so that $U=U_{c_{2}}$ represents a true zero-temperature critical point where the two solutions merge.

The other easily analyzable case is the noninteracting limit $U=0$. Here, a band-crossing transition takes place, where the density of states (DOS) at the Fermi level vanishes continuously and a gap opens at $t_{a b}=t$. The origin of this transition is easy to understand: in the atomic limit $t_{a b}>t$ the DOS reduces to the two (bonding and antibonding) levels at $E_{ \pm}= \pm t_{a b}$. When the hopping $t$ increases, these atomic levels broaden into bands of width $\approx 2 t$, so that the gap closes when the two bands overlap, at $t_{a b}=t$.

\section{RESULTS}

While the limit of infinite dimensions simplifies the original problem considerably, solving the corresponding impurity model is still a formidable task. In the framework of the single-band Hubpard megel, a number of numerically exact technique 111 $13,23,24$, as well as simpler approximate methods 12 . 12 . 13 have been used. A particularly simple approach first proposed by Georges and Kotliar 10 is based on solving the Anderson impurity model using second order merturbation theory, following Yamada and Yosida25 27. Due to the additional self-consistency this approach, often called the "iterated perturbation theory" (IPT) 14 , still has non-perturbative character. It is exact in both limits of $U=0$ and $U=\infty$ and displays a Mott metal-insulator transition. Detailed investigations based on other numerical approaches demonstrated 24,23 the qualitative validity of most IPT predictions for the single-band Hubbard model in $d=\infty$. As compared to numerically exact solutions, IPT requires considerably less computational effort, and thus represents a valuable guide to the physics of $d=\infty$ electrons.

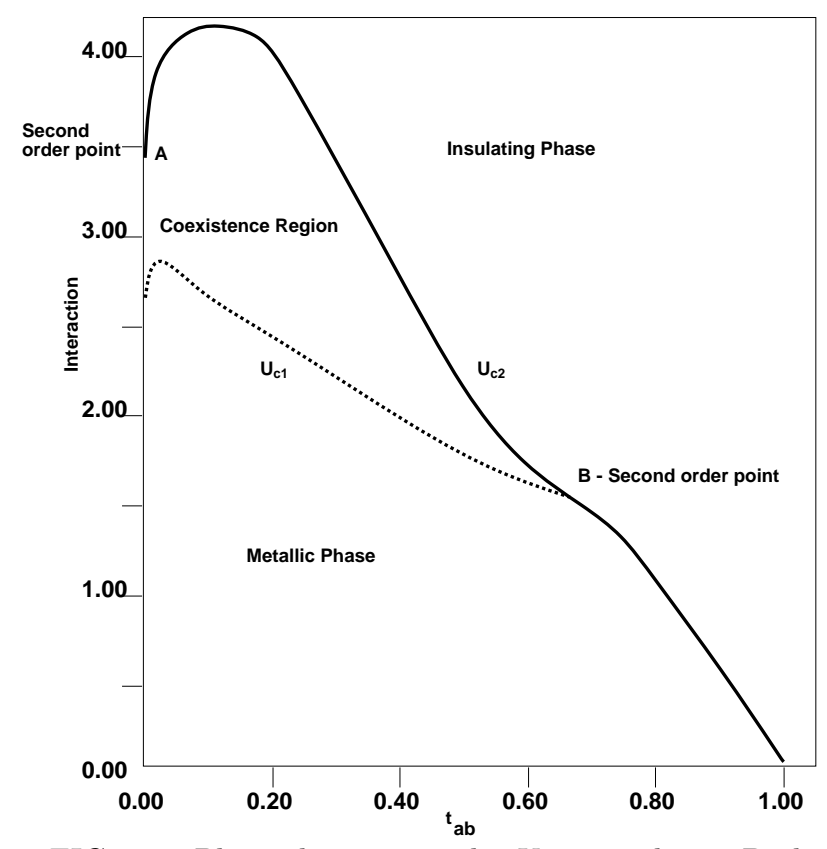

FIG. 2. Phase diagram in the $U-t_{a b}$ plane. Both the metallic and the insulating solution are locally stable in the coexistence region even at $T=0$, in contrast to the $t_{a b}=0$ situation. The curves denoted by $U_{c 1}$ (dashed line)and $U_{c 2}$ represent the boundaries (spinodals) of the insulating and the metallic solution, respectively. The two solutions merge at the two second order (critical) points denoted by $A$ and $B$. A direct, continuous transition from metal to insulator is found along the critical line (to the right of point B), which is qualitatively similar as for $U=0$ (see Figs. 3 and 4 ).

In the problem that we consider in this paper, one has to solve a two impurity Anderson model - a task which is considerably more difficult than the simpler one impurity model. Furthermore, numerical Monte Carlo approaches 28 to the two impurity Anderson model have proven to be largely unsuccessful at the available computational level. Alternative, exact diagonalization approaches also appear hardly feasible in the case of the two impurity problem, although recent developments29 hold considerable promise for the near future. Taking these facts into consideration, we propose to begin the investigation of the problem considered using the IPT approach as a useful first attempt to gain insight into the RKKYKondo competition. We note however, that in contrast to the earlier application of the IPT approach, in the present case this approximation is not exact in the $U=\infty$ limit, even at half filling. Still, we do not expect that these limitations will qualitatively modify our conclusions, especially in view of the absence of a small energy scale at the first order metal-insulator transition that we find. 
The possible instances where the limitations of the IPT approach could be relevant will be further discussed in Section IV, where we also present a quantitative estimate for the range of its validity.

In the following, we will concentrate on the behavior at half-filling, where the Mott transition takes place at $t_{a b}=0$, and investigate the modifications induced by turning on $t_{a b} \neq 0$. In order to apply IPT to the present model, we have to compute the second order perturbation theory corrections around the non-magnetic HartreeFock solution. The second-order diagonal/off-diagonal self-energies in this case consist of only one diagram respectively, and are given as

$$
\Sigma_{x y}(\tau)=-U^{2} G_{x y}^{0}(\tau) G_{x y}^{0}(-\tau) G_{x y}^{0}(\tau)
$$

where $x, y=a, b$. Since the resulting equations have to be solved self-consistently, the solution is obtained by numerical iteration until convergence is found.
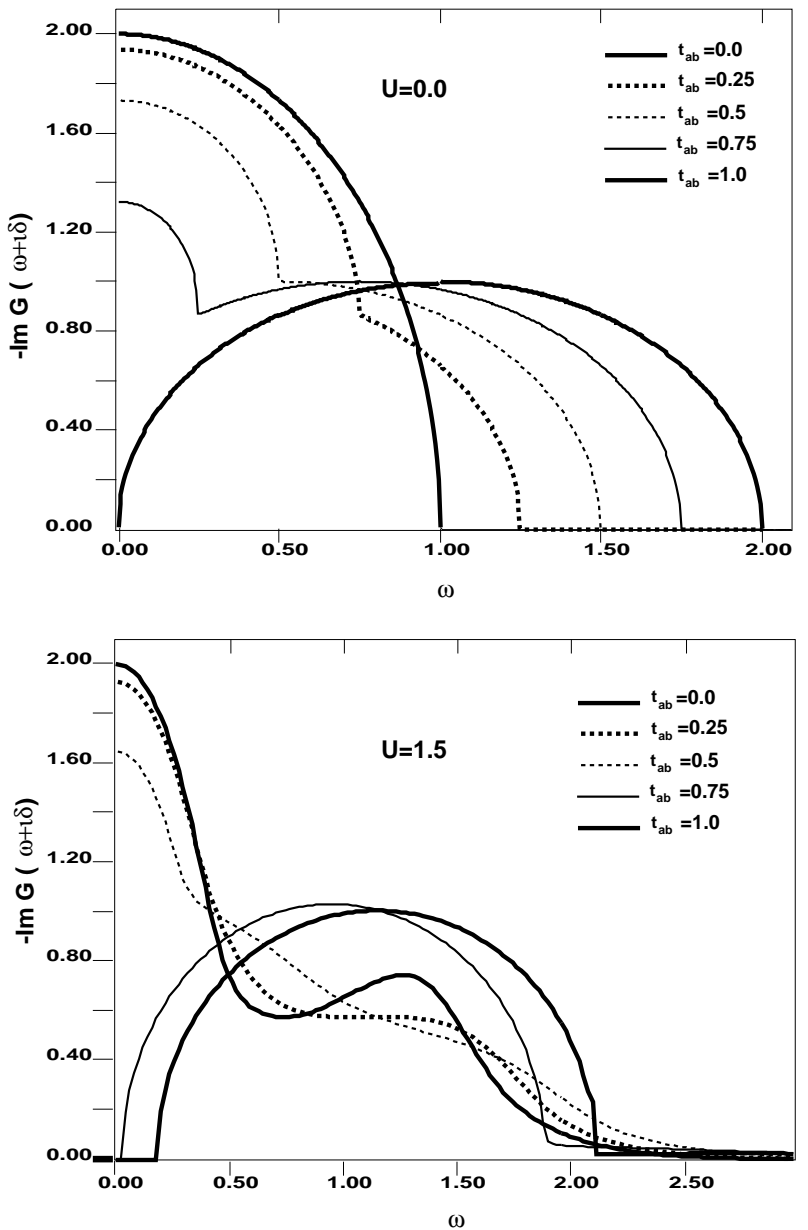

FIG. 3. Density of states for interactions a) $U=0$ and b) $U=1.5$ for $t_{a b}=0.0,0.25,0.5,0.75,1.0$.

We have determined the $T=0$ phase diagram of the investigated model at half filling using the IPT approximation, and the results are presented in Fig. (2). At small values of $U$, as $t_{a b}$ is increased, the behavior is qualitatively the same as at $U=0$. A continuous transition takes place, at a critical value of the hopping $t_{a b}^{c}(U)$ that is found to decrease as $U$ increases. This behavior reflects the fact that the gradual band broadening due to the Hubbard-Mott splitting tends to close the gap. As an illustration we display the evolution of the DOS as the transition is approached in Fig. (3) at $U=0$ (Fig.3(a)) and $U=1.5$ (Fig. 3(b)). [Throughout the paper, all energies are measured in units of the halfbandwidth $D=2 t]$

a)

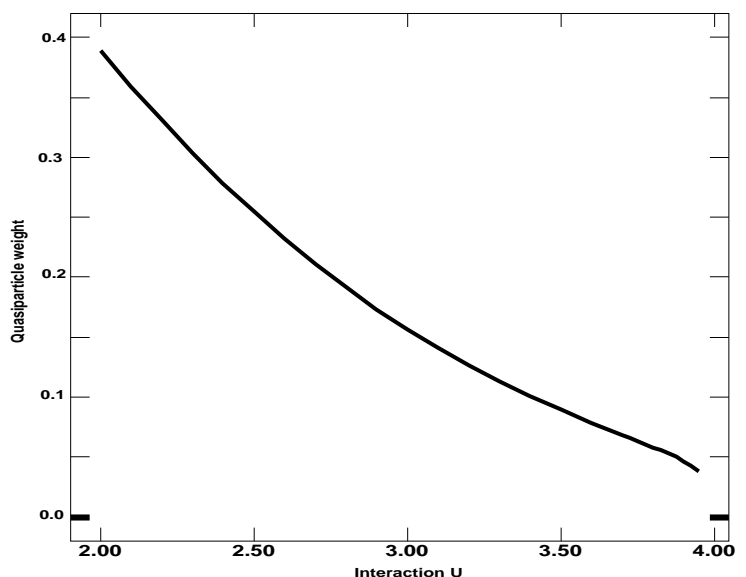

b)

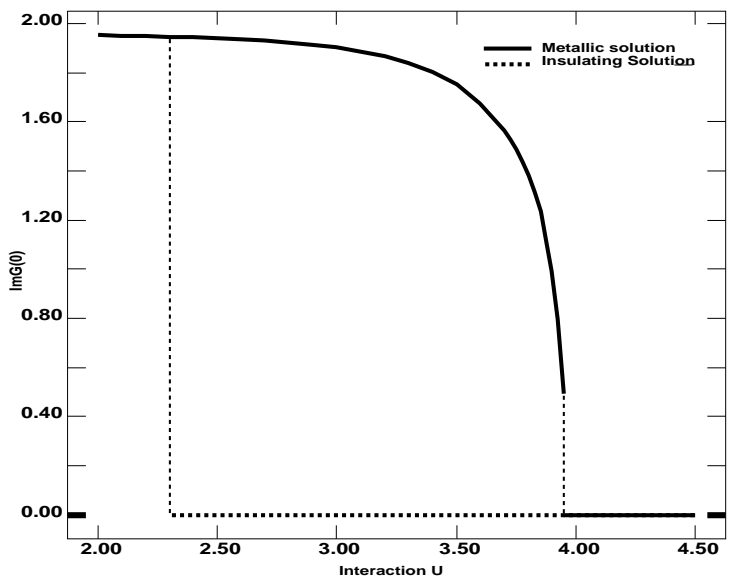

FIG. 4. a) Quasiparticle residue $z=1 /\left(1-\frac{\partial \Sigma}{\partial \omega}\right)$ in the metallic phase as a function of the interaction $U$ for $t_{a b}=0.2$. b) Density of States $\left(-\operatorname{Im} G\left(0^{+}\right)\right)$of metallic (solid line) and insulating (dotted line) solutions at $t_{a b}=0.2$ as a function of the interaction $U$.

For larger values of $U$, a coexistence region of the metallic and insulating solutions is found, similarly as for $t_{a b}=0$. The metallic solution is found for $U<U_{c_{2}}\left(t_{a b}\right)$; we note the non-monotonic dependence of $U_{c_{2}}\left(t_{a b}\right)$, which is first found to increase, and then to decrease as a function of $t_{a b}$. Thus, as compared with $t_{a b}=0$, the addition of the RKKY correlation is found to extend the metallic region. More importantly, we find that the metallic solution disappears discontinuously at $U=U_{c_{2}}\left(t_{a b}\right)$, in contrast to the $t_{a b}=0$ behavion 12.13. The solution along this boundary is not characterized by a low energy scale, 
as seen by plotting the Kondo temperature (quasiparticle weight $\left.z=1 /\left(1-\frac{\partial \Sigma}{\partial t}\right)\right)$ on one site as a function of $U$, for $t_{a b}=0.2$ (Fig. 1 (a)). The density of states is also discontinuous at this boundary (Fig.4(b)). We note the pronounced dependence of the density of states at the Fermi energy on the interaction $U-$ a clear violation of the "pinning condition" that is observed at $t_{a b}=0$. This behavior is seen even more clearly by plotting the evolution of the metallic DOS as the boundary is approached in Fig. (5). Clearly, in contrast to the situation at $t_{a b}=0$, the metallic and insulating solutions $d o$ not merge at $U=U_{c_{2}}\left(t_{a b}\right)$, so this boundary cannot be identified with a critical line.

Similar behavior is obtained by examining the stability of the insulating solution, which is found to discontinuously disappear at $U=U_{c_{1}}\left(t_{a b}\right)$. As we can see from the phase diagram, Fig. (2), the boundaries $U_{c_{1}}\left(t_{a b}\right)$ and $U_{c_{2}}\left(t_{a b}\right)$ are found to join at the critical point " $\mathrm{B}$ " which is also the end of the band crossing transition critical line.

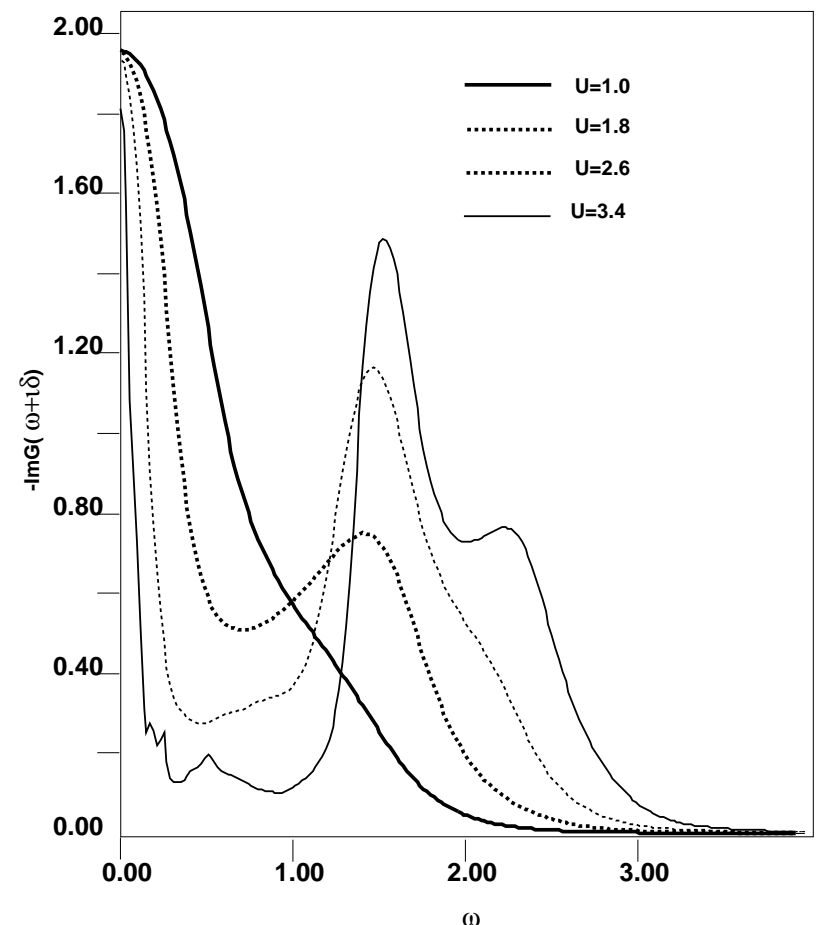

FIG. 5. Density of States for $t_{a b}=0.2$ and interactions $U=1.0,1.8,2.6,3.4$.

We thus find that in the entire coexistence region, the metallic and the insulating solutions merge only at two points: $\mathrm{A}\left(t_{a b}=0, U=U_{c_{2}}\right)$ and $\mathrm{B}$. In the rest of the phase diagram the two solutions are disjoint from each other, and the transition has a first-order character. This conclusion can be established even more rigorously by examining the local stability of each solution throughout the coexistence region. For this purpose, we have developed an approach that allows us to determine the stability, as described in detail in the appendix. Using this method, we have established that both the metallic and the insulating solution are locally stable, supporting the first-order scenario. (Note that when the procedure is applied in the $t_{a b}=0$ limit, we find that in the coexistence region, the insulating solution is locally unstable with respect to the metallic solution, in agreement with well established results 13.23 ).

To obtain the location of the transition line, we have calculated the energies of the solutions, and determined the line where they coincide, as shown in Fig. (2). Since the solutions merge at points A and B (see Fig. (2)), the energies of the solutions have to coincide there, and the (first order) transition line connects those two points. As an illustration, the energies of the two solutions are plotted for $t_{a b}=0.2$ as a function of $U$ in Fig. (6). As we can see, in contrast to the $t_{a b}=0$ findings, the insulating solution is lower in energy for larger values of $U$, consistent with the first-order scenario. This result is perhaps not surprising, as the RKKY interactions are generally expected to stabilize the insulating solution.

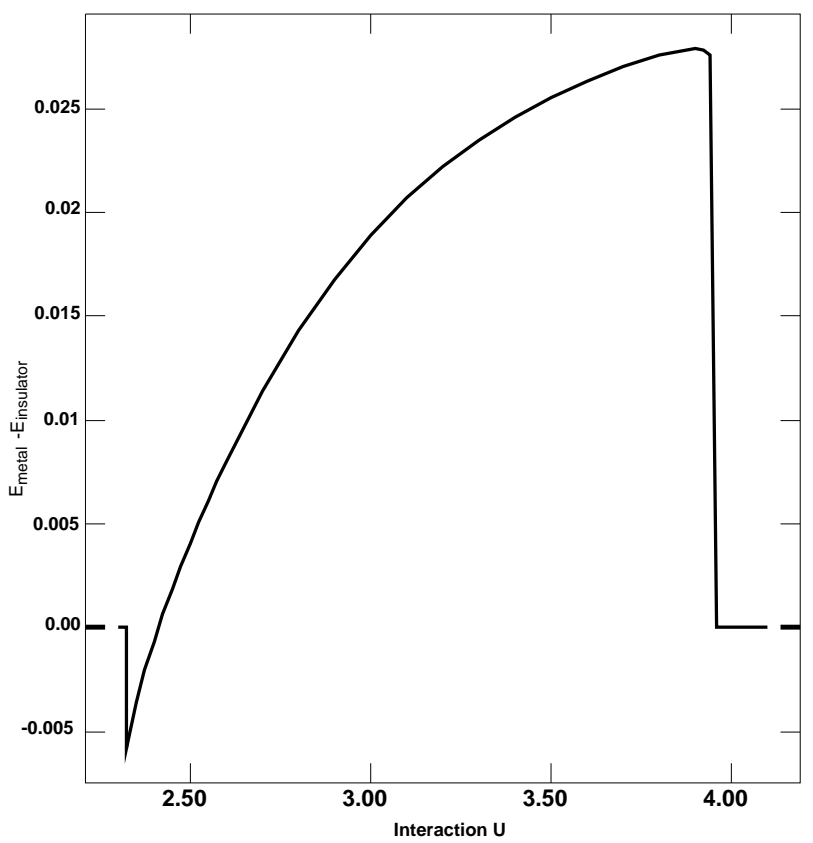

FIG. 6. Difference between the energies of metallic and insulating solutions for $t_{a b}=0.2$ as a function of the interaction $U$.

In line with this first order scenario, the boundary lines $U_{c_{1}}\left(t_{a b}\right)$ and $U_{c_{2}}\left(t_{a b}\right)$ should be recognized as spinodal lines. An interesting question is why the two solutions merge at $t_{a b}=0$, i. e. why is there a critical point there instead of a first-order transition. The existence of bifurcations, i. e. critical points is usually associated with spontaneous breakdown of some symmetry (e.g. up-down symmetry in the Ising model). In the case of the singleband Hubbard model in $d=\infty$ the relevant symmetry remains yet to be discovered.

The effects of the RKKY interactions are not limited to the modifications of the MIT scenario. They can also modify the thermodynamic behavior by affecting the dy- 
namics of the collective spin fluctuations governing the finite temperature response. In order to investigate this aspect of the problem, we have computed the specific heat in the metallic and the insulating phases of our model. To illustrate the typical metallic behavior, we present results for the specific heat at $U=2.5$, for three different values of $t_{a b}=0,0.2,0.4$ in Fig. (7).

At $t_{a b}=0$ we recognize the characteristic linear specific heat at $T<<T_{\text {Kondo }} \sim 0.05$, corresponding to Fermi liquid behavior, a Schottky-like peak at $T \sim$ $T_{\text {Kondo }}$ reflecting the binding energy of the Kondo singlet, and insulating-like behavior at $T \sim U / 2$ due to charge fluctuations (Hubbard bands).

For $t_{a b} \neq 0$, i.e. as the RKKY interactions are introduced, a new feature appears in the intermediate energy range: the specific heat is enhanced at $T \sim J_{a b}$, reflecting the emergence of additional spin fluctuations with a characteristic energy corresponding to the RKKY exchange interaction $J_{a b} \approx 4 t_{a b}^{2} / U$. For $t_{a b}=0.4$ and $U=2$ we estimate $J_{a b} \approx 0.08$, giving a ("Schottky") peak in the specific heat at $T_{J} \sim J / 2 \approx 0.16$, exactly where the observed enhancement occurs.

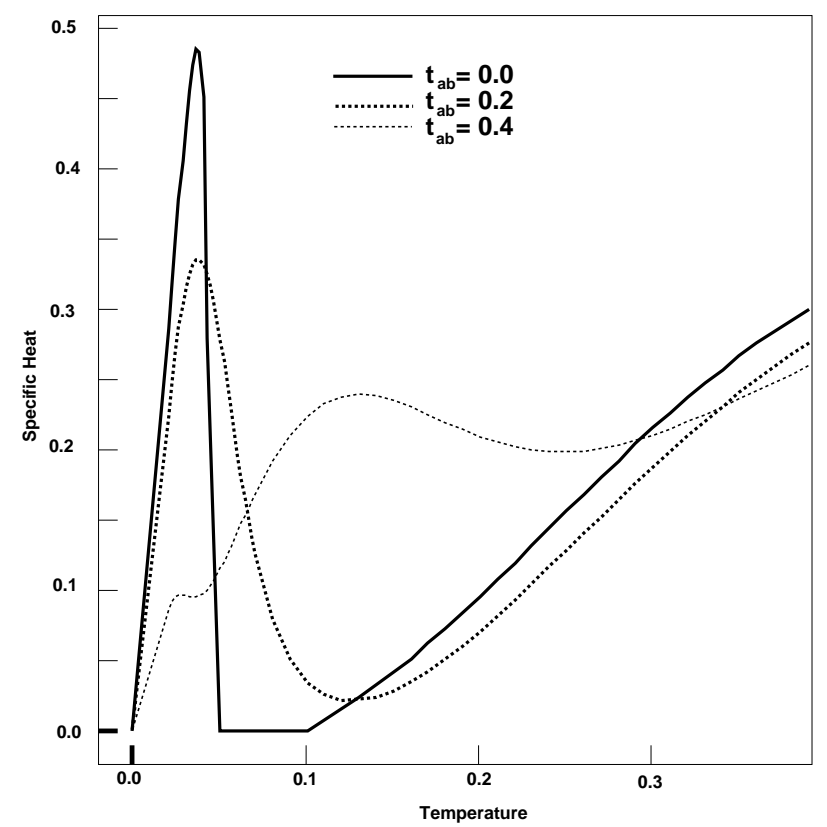

FIG. 7. Specific heat as a function of temperature for the metallic solution, at $\mathrm{U}=2.5$ for $t_{a b}=0.0,0.2,0.4$.

This interpretation of the observed specific heat enhancement finds additional support by examining the corresponding behavior in the insulating regime. Typical results are presented in Fig. (8), where the specific heat is plotted for $U=3.5$, and $t_{a b}=0,0.15$ and 0.3 .

In absence of RKKY interactions $\left(t_{a b}=0\right)$ the specific heat is vanishingly small at low temperatures, reflecting the existence of the Mott-Hubbard gap. As in the metallic phase, the addition of RKKY interactions $\left(t_{a b} \neq 0\right)$ induces specific heat enhancements in a comparable temperature range, at $T_{J} \approx 2 t_{a b}^{2} / U$. We also note that the corresponding enhancement in the metallic state (Fig. 7) is much more spread-out in temperature, presumably reflecting the scattering of these fluctuations by the coupling to the particle-hole excitations.

\section{BEYOND PERTURBATION THEORY}

The solution of our model presented in the preceding section was based on an approximate scheme for the impurity problem - theperturbation theory approach of Yosida and Yamada25 (YY). While this techniques was utilized with impressive success in previous $d=\infty$ studies1.14,22, it is important to emphasize the limitations of this approach, and identify instances where most important problems can be expected.

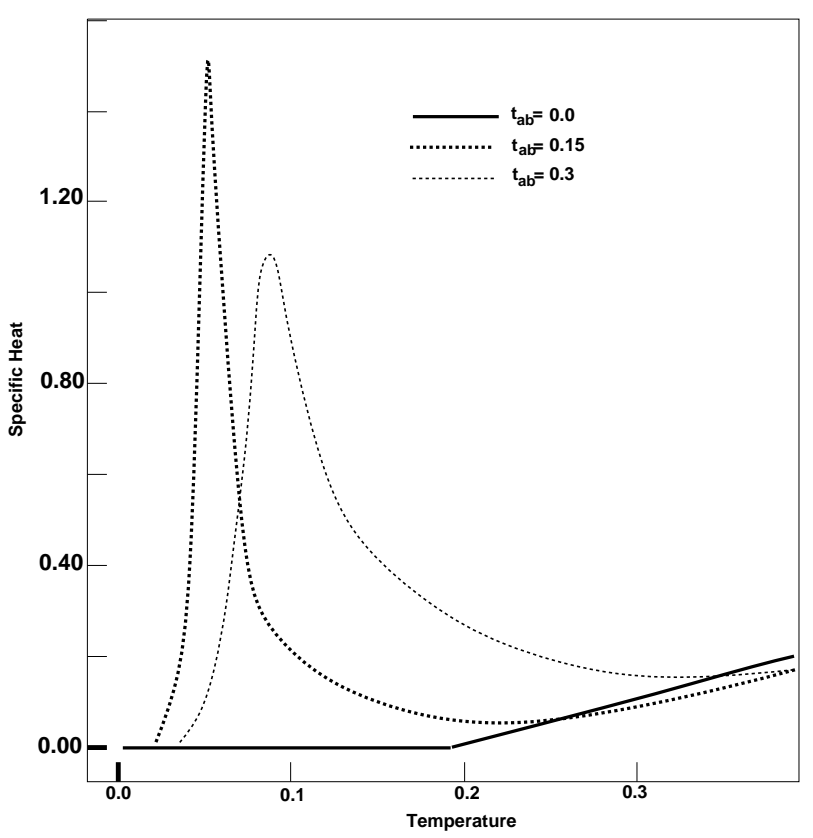

FIG. 8. Specific heat as a function of temperature for insulating solution, at $\mathrm{U}=3.5$ for $t_{a b}=0.0,0.15,0.3$.

When applied to single-impurity Anderson models, the approach of YY is generally expected to be at least qualitatively correct in the entire temperature range. In this case, the ground state is a (local) Fermi liquid, so that perturbation theory converges 32 and finite order corrections are sufficient. The situation is more complicated in two-impurity models such as the two-imfurity Kondo (Anderson) model. Here, a critical point 19 is found at half-filling, separating the RKKY and Kondo regimes. The emergence of this critical point has a simple physical origin. It reflects the fact that two ground states with different symmetry are possible, corresponding to the Kondo spins being compensated either by conduction electrons (Kondo phase) or by each other (RKKY phase). This critical foint, which reflects a degeneracy due to level crossing 30 signals a breakdown of a Fermi liquid description. As a result, we do not expect pertur- 
bative approaches to be accurate in the critical region. Indeed, if the two-impurity problem is treated in perturbation theory, the critical point is washed out into a smooth crossover.

Without providing a more elaborate treatment of the two-impurity problem, we can at least make estimates of the regions in parameter space where IPT could prove insufficient. Based on the information awailable from studies of the two impurity Kondo mode 19 in a fixed bath, we expect that a critical point emerges when the RKKY interaction $J_{a b}$ is comparable to the "bare" Kondo temperature $T_{\text {Kondo }}^{o}=T_{K o n d o}\left(t_{a b}=0\right)$. Since near $U_{c_{2}}$ the Kondo temperature vanishes

$$
T_{\text {Kondo }}^{o} \sim \frac{m_{o}}{m^{*}} \sim\left(U_{c_{2}}-U\right),
$$

but the exchange interaction remains finite

$$
J_{a b} \sim t_{a b}^{2} / U
$$

one can expect that increasing $U$ at finite $t_{a b}$ drives the system from a Kondo to an RKKY metallic phase. The critical line where this could take place can be estimated by equating $T_{K o n d o}^{o}$ and $J_{a b}$, and using Eqs. (7)-(8), we find

$$
U_{R K K Y}\left(t_{a b}\right) \approx U_{c_{2}}-4 t_{a b}^{2} / U_{c_{2}} .
$$

This expression is valid only in the $t_{a b} \rightarrow 0$ limit, where to leading order we have ignored the modifications of the (self-consistently adapting) electronic bath. This estimate is plotted in Fig. (9), where it is compared with the perturbation theory predictions for the metallic phase boundary, and the location of the first-order metal-insulator transition.

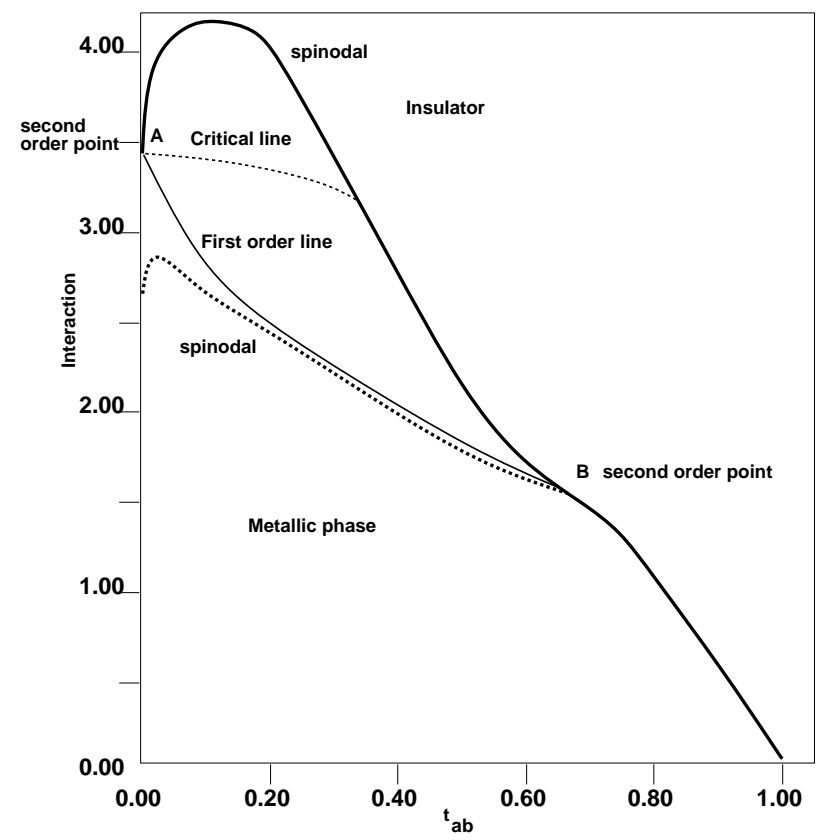

FIG. 9. Phase diagram indicating the critical line corresponding to the Kondo-RKKY phase transition. Also shown is the first order line found in our model.
As we can see, according to IPT, the first order transition preempts the approach to the RKKY-Kondo critical line, supporting the validity of IPT-based predictions.

The ITP prediction that the introduction of RKKY interactions induces a first-order metal insulator transition finds additional support if we recall that a similar conclusion was obtained by introducing additional RKKY interactions in the large- $\mathrm{N}$ approaches to correlated electrons 31 . However, we emphasize that this approach did not have the two impurity Kondo physics build in, and, in particular, the possibility that the RKKY-Kondo competition induces a nontrivial critical point even on the impurity level.

An interesting question that deserves further study is the role of the two impurity Kondo model critical point in the destruction of the metallic phase. Of course, this question would be particularly relevant if additions of small perturbations, perhaps disorder, could stabilize the metallic phase to larger values of $U$, so that the relevant critical point becomes physically accessible.

\section{CONCLUSIONS}

In this paper, we addressed the role of short-ranged magnetic correlations in determining the behavior of strongly correlated electronic systems. To account for these effect, which are not properly treated by existing approaches, we propose a two-site cluster generalization of the Hubbard model in infinite dimensions as the simplest model containing the relevant physics. The model is mapped onto a two-impurity Kondo-Anderson model in a self-consistently determined bath, making it possible to directly address the competition between the Kondo effect and RKKY interactions in a lattice context.

Using a well known approximation scheme for solving the self-consistency conditions, we have determined the phase diagram of our model and discussed the modifications of the metallic behavior. We find that the addition of RKKY interactions induces a first-order metalinsulator transition, by energetically favoring the insulating phase. Additional low-energy spin fluctuations emerge, leading to enhancements of the specific heat in the intermediate temperature range, both in the metallic and insulating phases.

\section{APPENDIX: STABILITY OF $d=\infty$ SOLUTIONS}

In this appendix, we describe a method that can be used to examine the local stability of the $d=\infty$ selfconsistency equations, Eqs. (2)-(5). The method is based on the observation that these equations can be derived using a variational approach, i.e. by extremizing a certain functional in analogy with standard Landau-Ginsburg formulations of mean-field theory.

In the present case, this functional takes a form 
$F\left[\mathbf{G}_{\sigma}\left(i \omega_{n}\right)\right]=-\frac{1}{2} t^{2} \frac{1}{\beta} \sum_{\sigma} \sum_{\omega_{n}} \operatorname{tr}\left[\mathbf{G}_{\sigma}^{2}\left(\omega_{n}\right)\right]+F_{i m p}\left[\mathbf{G}_{\sigma}\left(i \omega_{n}\right)\right]$

where $F_{i m p}\left[\mathbf{G}_{\sigma}\left(i \omega_{n}\right)\right]$ is the free energy of the twoimpurity model as defined by the action of Eqs. (4) and (5)

$$
F_{i m p}\left[\mathbf{G}_{\sigma}\left(i \omega_{n}\right)\right]=-\frac{1}{\beta} \ln \int D c^{*} D c e^{-S_{e f f}\left[\mathbf{G}_{\sigma}\left(i \omega_{n}\right)\right]} .
$$

Here, we consider Eqs. (4)-(5) as a definition of the effective action, so that $F\left[\mathbf{G}_{\sigma}\left(i \omega_{n}\right)\right]$ is a functional of a arbitrary, yet unspecified function $\mathbf{G}_{\sigma}\left(i \omega_{n}\right)$.

In analogy with conventional Landau-Ginsburg formulation, we think of $\mathbf{G}_{\sigma}\left(i \omega_{n}\right)$ as an order parameter (function). The mean-field equations are then obtained by extremizing the above functional with respect to variations in the form of $\mathbf{G}_{\sigma}\left(i \omega_{n}\right)$. The extremum condition reads

$$
\frac{\delta F[\mathbf{G}]}{\delta \mathbf{G}_{\sigma}\left(i \omega_{n}\right)}=0
$$

giving

$$
\mathbf{G}_{\sigma}\left(i \omega_{n}\right)=\mathbf{G}_{\sigma}^{i m p}\left(i \omega_{n}\right) .
$$

Here, $\mathbf{G}_{\sigma}^{i m p}\left(i \omega_{n}\right)$ is the local Green function of the impurity model corresponding to a fixed "bath" Green function $\mathbf{G}_{\sigma}\left(i \omega_{n}\right)$

$$
\mathbf{G}_{\sigma}^{i m p}\left(i \omega_{n}\right)=\left\langle c_{\sigma}^{*}\left(i \omega_{n}\right) c_{\sigma}\left(i \omega_{n}\right)\right\rangle_{S_{e f f}[G]} .
$$

Note that $\mathbf{G}_{\sigma}^{i m p}\left(i \omega_{n}\right)$ is also a functional of $\mathbf{G}_{\sigma}\left(i \omega_{n}\right)$.

Obviously, Eq. (14) is identical to Eq. (3), so that we recover the $d=\infty$ the self-consistency conditions Eq. (2)-(5).

Before going further, it is worthwhile to comment on the physical interpretation of the functional of Eqs. (10)(11). Using the expressions for the free energy of $d=\infty$ models 8 , one can show that our functional reduces to the free energy, when evaluated for the value of $\mathbf{G}_{\sigma}\left(i \omega_{n}\right)$ corresponding to the solutions of the self-consistency conditions, Eqs. (2)-(5). We conclude that $F[\mathbf{G}]$ represents a free energy functional, in the usual Landau-Ginsburg sense.

When the self-consistency conditions are solved numerically, one typically makes an initial guess for $\mathbf{G}_{\sigma}\left(i \omega_{n}\right)$, defining the effective action of the impurity model using Eq. (5). The impurity model is then solved by any method available, and a new value of $\mathbf{G}_{\sigma}\left(i \omega_{n}\right)$ obtained from Eq. (3). In numerical analysis, this iterative procedure is known as the "substitution-iteration method", which is repeated until convergence is reached. In the following, we prove a general theorem that such an iterative procedure converges towards a local (nearest) minimum of the free energy functional. We note that the set of all possible functions $\mathbf{G}_{\sigma}\left(i \omega_{n}\right)$ form a vector space (more precisely an infinite dimensional Hilbert space), and for notational simplicity, denote these vectors by $\mathbf{x}$.

We define the gradient vector field $\mathbf{g}(\mathbf{x})$ as

$$
\mathbf{g}(\mathbf{x})=\partial_{\mathbf{x}} F[\mathbf{x}]
$$

so that Eq. (12) can be written as

$$
\left.\mathbf{g}(\mathbf{x})\right|_{\mathbf{x}=\mathbf{x}_{o}}=0 .
$$

Here, $\mathbf{x}_{o}$ corresponds to the solution of the selfconsistency condition, i.e. is a locally stationary point of $F[\mathbf{x}]$. If we define further the quantity

$$
\mathbf{f}=\mathbf{x}-\mathbf{g}(\mathbf{x})
$$

we find that at the stationary point

$$
\mathbf{x}_{o}=\mathbf{f}\left(\mathbf{x}_{o}\right)
$$

In this language, the "substitution-iteration' search for the solution can be written as

$$
\mathbf{x}(n+1)=\mathbf{f}(\mathbf{x}(n)),
$$

and the solution corresponds to

$$
\mathbf{x}_{o}=\lim _{n \rightarrow \infty} \mathbf{x}(n)
$$

Note that the increment of $\mathbf{x}(n)$ can be also written as

$$
\Delta \mathbf{x}(n)=\mathbf{x}(n+1)-\mathbf{x}(n)=-\mathbf{g}(\mathbf{x}(n)) .
$$

As we can see, the iteration takes the vector $\mathbf{x}$ in the direction opposite to the gradient, i. e. "down the hill" , so that the iteration converges only in the vicinity of a local minimum.

On general grounds, we expect the physical solutions near first-order transitions to be locally stable. We can check this stability, by making a small modification in the initial conditions that produce the respective solutions. More precisely, we should first find the convergent metallic and insulating solutions $\mathbf{x}_{o}^{M}$ and $\mathbf{x}_{o}^{I}$. We can then examine the stability of, for example, the insulating solution by re-starting the iteration search from a new initial guess

$$
\mathbf{x}^{i}(n=0)=(1-c) \mathbf{x}_{o}^{I}+c \mathbf{x}_{o}^{M} .
$$

The solution is locally stable if for $c$ sufficiently small the iteration procedure converges to $\mathbf{x}=\mathbf{x}_{o}^{I}$. We can similarly check the stability of the metallic solution by choosing $c \approx 1$.

In order to apply these ideas, we first test them in the well examined limit corresponding to $t_{a b}=0$. We find that the metallic solution is stable throughout the coexistence region, but that the insulating solution becomes unstable as $T \rightarrow 0$. These findings are in complete agreement with the result 23 that $U_{c 2}$ is a $T=0$ critical point at which (upon reducing $U$ ) the insulating solution becomes unstable, and a new metallic solution emerges. We 
can apply these ideas for $t_{a b} \neq 0$, in which case both solutions are found to be locally stable, in agreement with a first-order scenario.

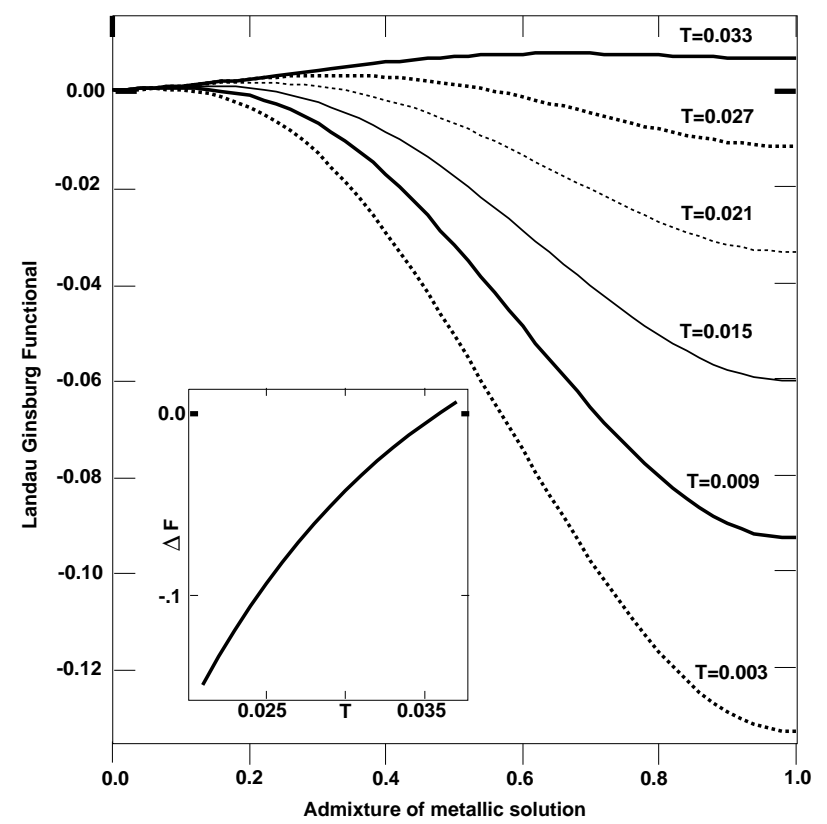

FIG. 10. Landau free energy for $t_{a b}=0, U=2.6$ and temperatures $T=0.003-0.033$ in steps of $\Delta T=0.006$. The inset shows the free energy difference between the two minima as a function of temperature.

We conclude this discussion by an explicit construction of the "Landau-Ginsburg" functional, which represents a nice illustration of the above stability considerations. To do this, we note that in the case of the $d=\infty$ equations, the gradient vector takes the form

$$
\mathbf{g}=\mathbf{G}^{i m p}[\mathbf{G}]-\mathbf{G},
$$

which can be calculated by any method that solyes the Anderson impurity model, e.g. the YY approach 25. Once the gradient is available, it is possible to determine the evolution of $F[\mathbf{x}]$ along any particular direction in the $\mathbf{x}$ space. In particular, we expect the physical solutions to be local minima, separated by an unstable solution (local maximum or a saddle-point). It is thus useful to consider the direction (vector) connecting the two solutions, which can be parameterized as

$$
\mathbf{x}(\ell)=(1-\ell) \mathbf{x}_{o}^{I}+\ell \mathbf{x}_{o}^{M} .
$$

The increment of $F[\mathbf{x}(\ell)]$ can be expressed as a line integral

$$
\Delta F(\ell)=F[\mathbf{x}(\ell)]-F\left[\mathbf{x}_{o}^{I}\right]=\int_{0}^{1} d \vec{\ell} \cdot \mathbf{g}(\mathbf{x}(\ell)) .
$$

We can numerically compute this line integral by an appropriate discretization procedure, and typical results in the $t_{a b}=0$ limit are plotted in Fig. (10).
Here, we show $\Delta F(\ell)$ for $U=2.6$ and for several different temperatures. As we can see, at $T \neq 0$ both the metallic and the insulating solution are locally stable, but the insulating one becomes unstable as $T \rightarrow 0$, in agreement with our stability considerations. We also note that as the temperature is increased, the free energy of the metallic solution $(\ell=1)$ increases, until the spinodal is reached, where the local minimum becomes an inflection point and becomes even locally unstable. Of course, this instability is preempted by a first order transition, which in this case happens finite temperature, in agreement with findings of Refs. 13.14 .

\section{ACKNOWLEDGMENTS}

We thank S. Barle, G. Kotliar, M. Rozenberg, and Q. Si for useful discussions. The work at Rutgers was supported by the NSF under grant DMR 92-24000 and the ONR under grant N-11378-RUCKENSTEIN. V. D. was supported by the Alfred P. Sloan Foundation, by the NSF under Cooperative Agreement No. DMR95-27035, and the State of Florida.

${ }^{1}$ For a review of heavy fermion materials see, for example: N. Grewe, and F. Steglich, in Handbook on Physics and Chemistry of Rare Earths, edited by K. A. Gschneidner, Jr. and L. Eyring (Elsevier Science Publishers B. V., 1991).

${ }^{2}$ P. Coleman and J. Gan, Physica B , 1991; J. Gan and P. Coleman, Phys. Rev. Lett. 68, 3476 (1992).

${ }^{3}$ For a recent review of doped semiconductors, see M. A. Paalanen, R. N. Bhatt, Physica B169, 231 (1991).

${ }^{4}$ R. N. Bhatt, D. S. Fisher, Phys. Rev. Lett. 68,3072 (1992).

${ }^{5}$ V. Dobrosavljević, T. R. Kirkpatrick, G. Kotliar, Phys. Rev. Lett. 69, 1113 (1992).

${ }^{6}$ M. A. Paalanen, J. E. Graebner, R. N. Bhatt, S. Sachdev, Phys. Rev. Lett. 61, 597 (1988).

${ }^{7}$ W. Metzner and D. Vollhardt, Phys. Rev. Lett. 62, 324 (1989).

${ }^{8}$ A. Georges, G. Kotliar, W. Krauth, and M. Rozenberg, Rev. Mod. Phys. 68, 13 (1996).

${ }^{9}$ W. L. Bragg and E. J. Williams, Proc. Roy. Soc. A 145, 699 (1934); see also K. Huang, Statistical Mechanics, (Wiley, New York, 1963).

${ }^{10}$ A. Georges and G. Kotliar, Phys. Rev. B45, 6479 (1992).

${ }^{11}$ M. Jarrel, Phys. Rev. Lett. 69, 168 (1992); Th. Pruschke, d. L. Cox and M. Jarrel, Europhys. Lett. 21, 5 (1993); Phys. Rev. B 47, 3553 (1993).

12 M. J. Rozenberg, X. Y. Zhang, and G. Kotliar, Phys. Rev. Lett. 691236 (1992).

${ }^{13}$ X. Y. Zhang, M. J. Rozenberg and G. Kotliar, Phys. Rev. Lett. 70, 1666 (1993).

14 A. Georges, W. Krauth, Phys. Rev. Lett. 69, 1240 (1992); Phys. Rev. B 48, 7167 (1993). 
${ }^{15}$ Q. Si and G. Kotliar, Phys. Rev. Lett. 70, 3467 (1993), Phys. Rev. B 48, 13881 (1993).

${ }^{16}$ E. Müller-Hartmann. Z. Phys. B, 74:507, 1989; Z. Phys. B, 76:211, 1989.

${ }^{17}$ V. Dobrosavljević and G. Kotliar, Phys. Rev. Lett. 71, 3218 (1993); Phys. Rev. B 50, 1430 (1994).

18 A. Schiller and K. Ingersent, Phys. Rev. Lett. 75, 113 (1995); A. Georges and G. Kotliar (unpublished); M. H. Hettler et al., preprint cond-mat/9803295.

19 B. A. Jones, C. M. Varma, and J. W. Willkins, Phys. Rev. Lett, 61, 125 (1988); A. J. Millis, B. G. Kotliar, B. A. Jones, in Field Theories in Condensed matter Physics: A Workshop, ed. by Z. Tesanovic (Addison Wesley, New York, 1990).

${ }^{20}$ In recent work, Kotliar and Georges (private communication) have proposed a cluster $1 / d$ expansion of the $d=\infty$ mean-field theory, that can be interpreted in terms of one and two-impurity models. Although this theory cannot be considered to be a exactly solvable limit of a definite lattice model, it offers an elegant way of restoring the translational invariance in the cluster formulation. On physical as well as on technical grounds, we expect the results of such a formulation to be close to the findings presented in this paper.

${ }^{21}$ R. N. Bhatt, P. A. Lee, Phys. Rev. Lett. 48, 344 (1982); D. S. Fisher, Phys. Rev. Lett. 69, 534 (1992).

22 The essentially same model was investigated in the context bilayer systems by H. Monien, N. Elstner, and A. J. Millis, preprint cond-mat/9707051.

23 M. J. Rozenberg, G. Moeller, and G. Kotliar, Modern Physics Letters B 8, 535 (1994).

${ }^{24}$ M. Caffarel and W. Krauth. Phys. Rev. Lett. 72, 1545 (1994).

${ }^{25}$ K. Yoshida and K. Yamada. Prog. Theor. Phys., N46, 244 (1970).

${ }^{26}$ K. Yamada. Prog. Theor. Phys., 53, 970 (1975).

${ }^{27}$ K. Yoshida and K. Yamada. Prog. Theor. Phys., 53:1286 (1975).

${ }^{28}$ R. M. Fye, J. E. Hirsch, D. J. Scalapino, Phys. Rev. B 35, 4901 (1987).

29 G. Moeller, Qimiao Si, G. Kotliar, M. Rozenberg, and D. S. Fisher, Phys. Rev. Lett. 74, 2082 (1995).

30 J. W. Rasul and P. Schlottmann, Phys. Rev. Lett. 62, 1701 (1989); P. Schlottmann and J. W. Rasul, Physica B 163, 544 (1990); S. Barle and A. E. Ruckenstein (unpublished).

${ }^{31}$ M. Grilli, G. Kotliar, Phys. Rev. Lett. 64, 1170 (1990); C. Castellani, M. Grilli, G. Kotliar, Phys. Rev. B 43, 8000 (1991).

${ }^{32}$ V. Zlatić and B. Horvatić, Phys. Rev. B 28, 6904, (1983). 